\title{
Tratamiento multidisciplinario de la endometriosis profunda grave
}

\author{
Gonzalo Hugo Zapata ${ }^{1}$, Beatriz Videla Rivero ${ }^{2}$, Alejandro Moreira Grecco', \\ Leopoldo Videla Rivero ${ }^{3}$ \\ ${ }^{1}$ MAAC, MSACP. Médico de planta del Hospital de Clínicas "José de San Martin”. CABA, Argentina. \\ ${ }^{2}$ Miembro de SACIL. CABA, Argentina. \\ ${ }^{3}$ Miembro de SACIL, SOGIBA, AAGL, SLS, SIAEGI. CABA, Argentina.
}

\begin{abstract}
RESUMEN
Introducción: La endometriosis es una entidad clínica caracterizada por la presencia de tejido endometrial fuera del endometrio, siendo la afección rectocolónica más agresiva.

Diseño: Retrospectivo de grupo único.

Objetivos: Evaluar la factibilidad de las resecciones colorrectales, patología compleja que debe abordarse en forma multidisciplinaria y reportar los resultados obtenidos de más de 10 años de trabajo.

Material y métodos: Estudio descriptivo y retrospectivo de una serie de casos. En el periodo comprendido entre 2005 y a principios de 2017, se evaluaron 29 pacientes. La evaluación se realizó en forma multidisciplinaria.

Resultados: Hemos operado 171 endometriosis profundas. La edad media fue 34,04 años. Veintinueve necesitaron resección de colon, en 27 se realizó una resección anterior con anastomosis colorrectal y 2 sigmoidectomías. Las anastomosis fueron con sutura mecánica, 17 término-terminales colorrectales y 12 término-laterales. Veintiocho anastomosis fueron realizadas entre los 7 y $5 \mathrm{~cm}$ del margen anal, 1 fue realizada a $4 \mathrm{~cm}$ del margen anal. En este caso se confeccionó una colostomía transversa de protección. El tiempo operatorio medio fue 90 minutos (45-195). El índice de conversión fue del $15 \%$. Las complicaciones fueron hemoperitoneo, fístula anastomótica e infecciones de piel. La estadía institucional la media fue de 5 días.

Conclusiones: El tratamiento laparoscópico de esta enfermedad es factible, debido a la distorsión de la anatomía de la pelvis que esta genera, aunque las tendencias actuales enfatizan la necesidad del tratamiento de esta afección de forma mínimamente invasiva.
\end{abstract}

Palabras clave: Endometriosis; Laparoscopía; Tratamiento

\begin{abstract}
Introduction: Endometriosis is a clinical entity characterized by the presence of endometrial tissue outside the endometrium, being the most aggressive rectocolonic condition.

Design: Descriptive, retrospective case series study.

Objective: Evaluate the feasibility of colorectal resections in this complex pathology that must be approached in a multidisciplinary way and report the results obtained from more than 10 years of work.

Material and methods: In the period between 2005 and early 2017, 29 patients were evaluated in a multidisciplinary way. Results: One hundred and seventy-one patients were operated on for deep endometriosis, mean age 34 years. Twentynine patients required colon resection, 27 anterior resection and 2 sigmoidectomies. Seventeen end-to-end and 12 end- toside stapled anastomoses were performed. Twenty-eight anastomoses were performed between 7 and $5 \mathrm{~cm}$ from the anal margin and one at $4 \mathrm{~cm}$. In the latter, a protective transverse colostomy was made. The mean operative time was 90 (45-195) minutes. The conversion rate was $15 \%$. Complications were hemoperitoneum, anastomotic fistula, and wound infections. The average hospital stay was 5 days.

Conclusion: Laparoscopic treatment of this disease is feasible. Despite the distortion it generates in the anatomy of the pelvis, the current trend emphasizes the need for minimally invasive treatment.
\end{abstract}

Keywords: Endometriosis; Laparoscopy; Treatment

\section{INTRODUCCIÓN}

La endometriosis es una entidad clínica caracterizada por la presencia de tejido endometrial fuera del endometrio, siendo la afección rectocolónica más agresiva, sobre todo cuando se asocia al compromiso del tabique rectovaginal. El compromiso del recto causa en las pacientes dolor pélvico crónico, dispareunia, infertilidad, inclusive rectorra-

Los autores declaran ausencia de conflictos de interés.

Gonzalo Hugo Zapata

gonzalohzapata@yahoo.com.ar

Recibido: julio de 2019. Aceptado: junio de 2020 gia y muchas veces dolor evacuatorio.

El tratamiento laparoscópico de esta enfermedad es difícil, debido a la distorsión de la anatomía de la pelvis que esta genera, aunque las tendencias actuales enfatizan la necesidad del tratamiento de esta afección de forma mínimamente invasiva.

\section{OBJETIVOS}

Evaluar la factibilidad de las resecciones colorrectales en la endometriosis profunda, esta patología compleja que debe abordarse en forma multidisciplinaria y reportar los 
resultados obtenidos de más de 10 años de trabajo.

\section{MATERIALY MÉTODO}

Estudio descriptivo y retrospectivo de una serie de casos en un centro de derivación, en un periodo entre 2005 y principios de 2017. Se evaluaron 29 pacientes. La evaluación se realizó en forma multidisciplinaria. Las pacientes fueron siempre derivadas de otros centros, incluido el interior del país, para el tratamiento quirúrgico. Se consideraron criterios de inclusión pacientes con dolor pélvico crónico, diagnóstico de endometriosis profunda previo y pacientes con compromiso profundo colónico y de exclusión, las pacientes sin compromiso del colon por laparoscopía diagnóstica previa. El estudio preoperatorio se realizó con eco tv, resonancia magnética alta resolución y videocolonoscopía, se registró demografía de la muestra, antecedentes ginecológicos y de tratamiento de la endometriosis, hormonoterapia, cirugía realizada, tiempo operatorio, distancia de la anastomosis al margen anal, realización de ostomía de protección, estadía hospitalaria, morbi mortalidad. Para la evaluación de las complicaciones se utiliza la clasificación de Clavien- Dindo. ${ }^{1}$

\section{RESULTADOS}

En el periodo descrito, hemos operado 171. La edad media fue 34,04 años (rango 18-52) y el motivo de consulta, dolor pélvico crónico (tabla 1 ).

Veintinueve necesitaron resección de colon, en 27 se realizó una resección anterior con anastomosis colorrectal y 2 sigmoidectomías. Las anastomosis fueron con sutura mecánica, 17 término-terminales colorrectales y 12 términolaterales (a la cara anterior del recto medio). Veintiocho anastomosis fueron realizadas entre los 7 y $5 \mathrm{~cm}$ del margen anal, 1 fue realizada a $4 \mathrm{~cm}$ del margen anal. En este caso se confeccionó una colostomía transversa de protección.

El tiempo operatorio medio fue 90 minutos (45 minutos hasta 195 minutos). Todas las cirugías fueron realizadas por el equipo de Ginecología y Coloproctología y, en los casos de compromiso uretral, el equipo de Urología. El índice de conversión fue del 15\%. Los procedimientos asociados realizados por el equipo de Ginecología (tabla 2). Complicaciones (tabla 3).

La estadía institucional media fue de 5 días (entre 4 y 10 días).

\section{DISCUSIÓN}

Desde 1899, con los trabajos de Russell, la endometriosis se define como la "Presencia de tejido endometrial, glán-
TABLA 1: SÍNTOMAS

$\begin{array}{lcc}\text { Síntomas } & \text { Pacientes (n:29) } & \text { Porcentaje \% } \\ \text { Dispareunia } & 29 & 100 \\ \text { Dolor pélvico } & 29 & 100 \\ \text { crónico } & & \\ \text { Rectorragia } & 4 & 14 \\ \text { Infertilidad } & 10 & 34\end{array}$

\section{TABLA 2: PROCEDIMIENTOS}

\begin{tabular}{lc} 
Procedimiento & Número de pacientes \\
\hline Quistectomía ovárica & 12 \\
\hline Destechamiento & 14 \\
\hline Anexohisterectomía & 1 \\
\hline Anexectomía unilateral & 2
\end{tabular}

TABLA 3: MORBILIDAD SEGÚN LA CLASIFICACIÓN DE CLAVIEN-DINDO

\begin{tabular}{lcc} 
Tipo de complicación & $\begin{array}{c}\text { Número de } \\
\text { pacientes }\end{array}$ & $\begin{array}{c}\text { Clasificación } \\
\text { (Clavien-Dindo) }\end{array}$ \\
\hline Hemoperitoneo & 2 & IIIB \\
\hline Fístula anastomótica & 1 & IIIB \\
Infecciones de pared & 4 & II
\end{tabular}

dulas y estroma, fuera de la cavidad uterina". En la década de los 90, se define el término de endometriosis profunda para las lesiones que infiltran más de $5 \mathrm{~mm}$ afectando a los órganos subyacentes. ${ }^{2}$

Es una enfermedad ginecológica común que afecta aproximadamente al 10\% de las mujeres durante su edad reproductiva. Los costes anuales de endometriosis en asistencia sanitaria y los producidos por pérdida de la productividad se han estimado en 2801 y 1023 dólares por paciente, respectivamente. Estos costes son considerablemente más altos que los relacionados con la enfermedad de Crohn o con la jaqueca. ${ }^{2}$

En todas las mujeres un poco de la menstruación cae dentro de la cavidad abdominal a través de las trompas. Existen mecanismos de defensa que neutralizan esas células endometriales que caen en el abdomen. En algunas mujeres, por causas desconocidas, esos mecanismos fallan y las células se implantan fuera del útero, constituyendo la endometriosis en forma de implante, nódulo o quiste. En algunas ocasiones, la endometriosis puede localizarse en cicatrices quirúrgicas, los intestinos o el recto, la vejiga, la vagina, la vulva y el cuello del útero. Es una enfermedad benigna. La malignización es muy poco frecuente. Los implantes responden a las hormonas femeninas, produciendo inflamación, dolores y posteriormente cicatrices que adhieren los órganos entre sí, alterando la anatomía normal de la pelvis y su función reproductiva. ${ }^{3}$

Tiene en su etiopatogenia un importante componente genético, epigenético, de hormono-dependencia e infla- 
matorio; por lo que debe ser considerada como una enfermedad crónica (Tabla 4). ${ }^{4}$

Las teorías actuales son:

1. Menstruación retrógrada es la más aceptada, derivaría de un reflujo de tejido endometrial a través de las trompas de Falopio durante la menstruación y la implantación subsiguiente en el peritoneo de la pelvis y en el ovario;

2. implantación de células madre endometriales es una teoría derivada de la precedente, en el desarrollo de células endometriales, algunas células madres se dispersan por todo el peritoneo;

3. anormalidades del remanente mülleriano sugerida por la implantación en el fondo de saco de Douglas y en los ligamentos uterosacros, una diferenciación aberrante o migración del conducto de Müller puede ser la causa de la dispersión de células endometriales en la vida fetal;

4. metaplasia celómica el peritoneo que cubre la pelvis y los ovarios pueden tener metaplasia hacia células endometriales.

La endometriosis del tabique rectovaginal corresponde a la forma más severa. Puede infiltrar tanto la vagina como el recto y, en los casos más severos, puede extenderse lateralmente llegando a comprometer uno o ambos uréteres. E1 compromiso intestinal, aunque infrecuente, se observa en el 3,37\% de las pacientes con endometriosis y afecta principalmente el rectosigmoides. ${ }^{5,6}$ En nuestra serie, el 16\% de las pacientes operadas tenían compromiso colónico. Esta alta incidencia de compromiso colorrectal se ha evidenciado en otros centros de referencia para el tratamiento de la endometriosis. ${ }^{7}$

La definición del cuadro clínico característico para la endometriosis profunda con compromiso intestinal es difícil. Debe tenerse presente que se presenta principalmente con dolor pélvico (ej. dismenorrea, dispareunia profunda, algia pelviana no cíclica). Pueden coexistir síntomas gastrointestinales bajos y/o cambios en el hábito intestinal, secundarios al compromiso colorrectal, como son la rectorragia, el tenesmo rectal y la defecación dolorosa durante la menstruación. ${ }^{8-10}$ Pero más importante aún, es entender que el cuadro clínico con compromiso intestinal es muy variado, haciendo su diagnóstico difícil.

La utilidad del examen físico en el diagnóstico es motivo de controversia. Los hallazgos son variados y dependen de la localización de las lesiones y del día del ciclo menstrual en que se realice el examen. ${ }^{11,12} \mathrm{Si}$ bien lo más frecuente es palpar un nódulo sensible en el fondo del saco vaginal posterior, su ausencia no se descarta. Según Chapron et al., ${ }^{13}$ en un $65 \%$ de las pacientes con endometriosis profunda y compromiso rectal no se palpan nódulos dolorosos al tacto vaginal. La presencia de lesiones rojas duran-
TABLA 4: FISIOPATOLOGÍA ${ }^{4}$

Factores epidemiológicos

Factores reproductivos y menstruales:

Paridad $\downarrow \downarrow$

Edad de la menarca (temprana) $\uparrow$

Duración ciclo menstrual (short) $\uparrow$

Duración de las pérdidas $\uparrow$

Factores constitucionales:

Historia familiar $\uparrow$

Índice de masa corporal $\downarrow$

Pecas $\uparrow$

Nevos $\uparrow$

Hábitos personales:

Alcoholismo $\uparrow$

Dieta: inconsistente

Tabaquismo: sin efecto

Ejercicio regular $\downarrow$

Alteraciones celulares y moleculares

Biosíntesis de esteroides y respuesta del receptor alterada:

Expresión ERß aumentada

Expresión de aromatasa aumentada

Trastornos intermedios de la señal de progesterona: HOXA10, FOX01, NF-kß, Hlc-5, NCoR2

Deficiencia en 17-Bhydroxysteroid dehydrogenase-2

Mayor invasividad y vascularización:

Expresión no regulado MMP

BEGF peritoneal aumentado

AKT hiperactivo

Reclutamiento de la expresión de macrofagos Tie-2

Respuesta inflamatoria:

Producción de quemokinas: RANTES, MCP-1, IL-8

Reclutamiento de macrófagos activados alternativamente

Aumento peritoneal de IL-6, TNF

Compromiso de ruta dependiente de NF-k-B

Acumulación de hierro y producción de ROS

te la especuloscopía constituye otro hallazgo sugerente de endometriosis del TRV. Sin embargo, está ausente en el $70 \%$ de los casos de endometriosis intestinal. Con respecto al tacto rectovaginal, es claro que su utilidad es limitada, puesto que las lesiones habitualmente se ubican más altas quedando fuera del alcance. En base a nuestra experiencia, recomendamos fuertemente examinar a la paciente durante el periodo menstrual, si la anamnesis sugiere la presencia de endometriosis profunda.

Dentro de los exámenes complementarios, ultrasonografía transrectal para su correcta interpretación se requie- 
re de personal entrenado y de realización rutinaria. Otros han preconizado la ultrasonografía transvaginal, las imágenes aparecen como engrosamientos lineales o nódulos hipoecoicos de tamaño variable dependiendo del momento del ciclo menstrual. ${ }^{14}$ Bazot et al., ${ }^{15}$ en un estudio prospectivo controlado, incluyeron 142 pacientes y demostraron que era una herramienta útil en el diagnóstico del compromiso endometriósico intestinal. La presencia de endometriomas es un marcador de enfermedad severa y un factor de riesgo independiente para la necesidad de resección intestinal durante el tratamiento quirúrgico. En un análisis de 1785 pacientes con endometriosis ovárica, solo el 1\% tenía lesión única. ${ }^{16,17}$

Un aspecto a considerar se refiere al compromiso de otras estructuras pelvianas, particularmente al uréter. Habitualmente corresponde a un atrapamiento o estenosis secundaria a la fibrosis que lo compromete por vecindad. Afortunadamente tal compromiso es infrecuente. Sin embargo, en aquellos nódulos de gran tamaño $(\geq 3 \mathrm{~cm})$ y/o con extensión lateral, tal compromiso puede alcanzar el 11\% de los casos. El establecer su diagnóstico previo o durante la cirugía es de vital importancia pues, sin tratamiento y producto de la obstrucción, puede llevar a la pérdida de la función renal. Basado en lo anterior, es que hemos decidido, en nuestro centro, realizar estudio urológico a todas las pacientes con nódulo tipo III según la clasificación de Donnez (Tabla 5), los mayores de $3 \mathrm{~cm}$ o aquellos con extensión lateral. La instalación preoperatoria de catéteres doble J permite la identificación del uréter y facilita la ureterolisis. Aun así, el tratamiento quirúrgico es complejo y puede requerir de la resección parcial del uréter y el posterior reimplante en la vejiga.

La resonancia magnética nuclear (RM) constituye una herramienta diagnóstica cada vez más utilizada en la evaluación preoperatoria de la endometriosis profunda de la pelvis posterior. Esta técnica es superior a la ultrasonografía y entrega información más completa y objetiva de la anatomía de la pelvis. Sin embargo, la RM muestra al- gunas limitaciones con la presencia de endometriomas en la cercanía de los ligamentos uterosacros o con úteros en retroversión. ${ }^{17,18}$ En nuestra experiencia, la RM tiene gran correlación con los hallazgos intraoperatorios.

Con la videocolonoscopía se busca identificar lesiones con compromiso transmural intestinal y poder biopsiarlas. Sin embargo, el compromiso de la mucosa es infrecuente, por lo que habitualmente el examen resultará normal o con indicios de compresión extrínseca. ${ }^{19}$ En nuestra experiencia solo una de las 171 mujeres presentaba compromiso mucoso colónico.

Se ha comparado la sensibilidad de los diferentes métodos diagnósticos (Tabla 6).

Confirmado el diagnóstico, la única alternativa terapéutica efectiva en pacientes sintomáticas es lograr la resección quirúrgica completa del tejido endometrial ectópico. Sin embargo, tal cirugía es compleja y se asocia a posibles complicaciones. ${ }^{20-22}$ Por ende, la planificación previa y la coordinación en la participación de un equipo multidisciplinario es crucial.

La terapia médica, antes o después de la cirugía, se utiliza con el fin de facilitar el procedimiento o para disminuir el riesgo de recurrencia, aunque no existe evidencia que lo avale. Esta opción de tratamiento es solo transitoria y no debe constituir la alternativa de manejo definitivo. ${ }^{23}$

No existe consenso claro sobre las indicaciones con las cuales debe realizarse una resección intestinal en endometriosis, en general se indica en pacientes sintomáticas,

TABLA 5: CLASIFICACIÓN DE DONNEZ DE LOS NÓDULOS ENDOMETRIÓSICOS RETROPERITONEALES

\begin{tabular}{|c|c|}
\hline Tipo nódulo & Características \\
\hline Tipo I & $\begin{array}{l}\text { Tabique rectovaginal tamaño } 2 \text { cm (15\% } \\
\text { de los nódulos) }\end{array}$ \\
\hline Tipo II & $\begin{array}{l}\text { Ubicación retrocervical ( } 60 \% \text { de los nódu- } \\
\text { los) }\end{array}$ \\
\hline Tipo III & $\begin{array}{l}\text { "Reloj de arena" o Diabolo-Like tamaño } 3 \\
\text { cm infiltra pared rectal }\end{array}$ \\
\hline
\end{tabular}

TABLA 6: MÉTODOS DIAGNÓSTICOS

\begin{tabular}{|c|c|c|c|c|c|c|c|}
\hline Examen & $\begin{array}{l}\text { Sensibilidad } \\
\text { (\%) }\end{array}$ & $\begin{array}{c}\text { Especificidad } \\
(\%)\end{array}$ & $\begin{array}{l}\text { VPP } \\
(\%)\end{array}$ & $\begin{array}{l}\text { VPN } \\
(\%)\end{array}$ & $\operatorname{LR}(+)$ & $\operatorname{LR}(-)$ & "Gold estándar" \\
\hline Ustr (ultrasonografia transrectal) & 100 & 98 & 75 & 100 & 50 & - & Cx, histo \\
\hline $\begin{array}{l}\text { Ustv (ultrasonografia trasn- } \\
\text { vaginal) }\end{array}$ & 91 & 98 & 97 & 91 & 46 & 0.1 & Cx, histo \\
\hline Endosonografia & 92 & 84 & 85 & 89 & 5.8 & 0.1 & Cx, histo \\
\hline $\begin{array}{l}\text { Rmn (resonancia magnetica } \\
\text { nuclear) }\end{array}$ & 78 & 93 & 90 & 83 & 11 & 0.2 & Cx, histo \\
\hline Enema baritado & 100 & 98 & 98 & 100 & 50 & - & Cx, histo \\
\hline Tac (tomografía axial computada) & 99 & 100 & 100 & 93 & - & 0.01 & Histo \\
\hline
\end{tabular}


cuando existe sospecha de malignidad, ante la presencia de implantes profundos que comprometan $>50 \%$ del perímetro intestinal, con nódulos $>3 \mathrm{~cm}$, múltiples nódulos o con compromiso transmural. ${ }^{24-26}$

Desde la realización de la primera resección intestinal laparoscópica por endometriosis en $1991,{ }^{27}$ esta opción se ha transformado en una alternativa posible para el manejo de la endometriosis profunda de la pelvis posterior con compromiso intestinal. ${ }^{28,29}$

Existen varios tipos de resección en cirugía de la endometriosis profunda con compromiso colónico, la resección completa, la resección discoide y el shaving. ${ }^{30,31}$

La resección anterior laparoscópica ha reportado una tasa de éxito en el tratamiento de la endometriosis cercana al $94 \%$, con una morbilidad aceptable aproximada del 15\%. ${ }^{32}$

En una serie del grupo del Hospital Italiano de Buenos Aires, diecisiete pacientes fueron operadas por endometriosis profunda severa. ${ }^{31}$ La mayoría consultaba por dolor pélvico crónico, la edad media de las pacientes fue de 35 años, el lugar más comprometido fue el recto en un 52\% de los casos, seguido por la unión rectosigmoidea, en 9 pacientes se realizó resección anterior baja con 4 ostomías de protección, en 5 se realizó resección anterior alta y en $3 \mathrm{pa}-$ cientes hemicolectomía derecha. La morbilidad global fue del $23 \%$ con una dehiscencia anastomótica y una mortalidad nula.

Según una serie del grupo de Chapron, 100 mujeres con dolor pélvico crónico que fueron reoperadas, lo fueron por cirugías incompletas, por eso promueven la resección completa de la lesión, incluido el segmento colónico afectado. ${ }^{33}$

La tasa de recurrencia así fue del $2 \%$ y la tasa de fístulas en las anastomosis no protegidas en nuestra serie fue del $0,03 \%$, un caso de 28 . Impresiona que la incidencia de fístulas en este tipo de pacientes es menor que en las resecciones rectales por cáncer.

Las resecciones en disco representan una alternativa que limita la disección pélvica, resultado en un menor trauma quirúrgico y menor tiempo operatorio. Moawad et al., ${ }^{30}$ comparó la resección anterior con la resección discoide laparoscópica hecha en 8 pacientes, contra 14 resecciones anteriores, su trabajo reportó menor pérdida sanguí- nea, menores complicaciones asociadas y menor tiempo hospitalario. Pero en contrapartida, 2 tuvieron estenosis rectales que necesitaron dilataciones. En este reporte no menciona fístulas anastomóticas en ninguna de las dos opciones terapéuticas. Las lesiones que trataron eran menores de $3 \mathrm{~cm}$ de diámetro.

Como alternativa más sencilla se ha propuesto el shaving (o resección nodular) de la pared intestinal. La ventaja de la baja morbilidad cuando se realiza una resección nodular no está necesariamente relacionada con un aumento de la recurrencia del dolor si el nódulo recidiva, especialmente en mujeres que se pueden beneficiar con tratamiento médico posterior.

La serie de Renner et al. del 2017,32 informó la realización de shaving rectal con bisturí con ultrasonido o plasma en 64 y 58 mujeres, respectivamente. Los nódulos clasificados en $<1 \mathrm{~cm}$ y $>3 \mathrm{~cm}$ de diámetro, se localizaron en el recto medio y superior. Exceptuando dos fístulas rectales $(1,6 \%)$, la mayoría de las complicaciones no estaban relacionadas con el afeitado rectal. Los resultados revelaron una mejoría estadísticamente significativa en la función digestiva y en el dolor pélvico a los 1 y 3 años después del afeitado rectal, pero no el estreñimiento. Recidivas rectales ocurrieron en el $4 \%$ de los pacientes, el 2,4\% de los cuales tenían resección segmentaria, $0,8 \%$ afeitado y $0,8 \%$ escisión en disco.

\section{CONCLUSIONES}

Nuestros resultados muestran que el abordaje de la endometriosis profunda de la pelvis debe ser multidisciplinario, incluyendo ginecólogos, proctólogos, urólogos y especialistas en diagnóstico por imágenes.

La cirugía laparoscópica, aunque muchas veces engorrosa, es segura y se puede realizar si el equipo quirúrgico se encuentra familiarizado con esta patología desafiante. Bajo ningún concepto se debe tomar como un fracaso la conversión a laparotomía o continuar el procedimiento por la vía convencional luego del diagnóstico laparoscópico, lo que debe ser algo consensuado por el equipo tratante.

\section{BIBLIOGRAFÍA}

1. Dindo D, Demartines N, Claiven P. Classification of surgical complications. A new proposal with evaluation in a cohort of 6336 patients and results of a survey. Ann Surg 2004;240:205-13.

2. Vercellini P. Deep Endometriosis: Definition, pathogenesis and clinical management. JAAGL 2004;11:153-61.

3. Simoens S, Hummelshoj L, D囚 Hooghe T. Endometriosis: cost estimates and methodological perspective. Hum Reprod Update 2007;13:395-404.

4. Definición: ¿Qué es la Endometriosis? Sociedad Argentina de Endometriosis. https://saendometriosis.com.ar/practice-areas/
5. Vercellini P, Vigano P, Somigliana E, Fedele L. Endometriosis: pathogenesis and treatment. Nat Rev Endocrinol 2014;10:261-75.

6. Waller KG, Shaw RW. Gonadotropin-releasing hormone analogues for the treatment of endometriosis: long-term follow-up. Fertil Steril1993;59:511-15.

7. Sampson JA. Intestinal adenomas of endometrial type. Arch Surg1922;5:217-21.

8. Chapron C, Fauconnier A, Vieira M, Barakat H, Dousset B, Pansini $\mathrm{V}$, et al. Anatomical distribution of deeply infiltrating endometriosis: 
surgical implications and proposition for a classification. Hum Reprod 2003;18:157-61.

9. Cameron IC, Rogers S, Collins MC, Reed MW. Intestinal endometriosis: presentation, investigation, and surgical management. Int J Colorect Dis 995;10:83-6.

10. Adamson DG. Diagnosis and clinical presentation of endometriosis. Am J Obstet Gynecol 1990;162:568-69.

11. Chapron C, Barakat H, Fritel X, Dubuisson JB, Bréart G, Fauconnier A. Presurgical diagnosis of posterior deep infiltrating endometriosis based on a standardized questionnaire. Hum Reprod 2005;20:507-13.

12. Koninckx PR, Meuleman C, Oosterlynck D, Cornillie FJ. Diagnosis of deep endometriosis by clinical examination during menstruación and plasma CA-125 concentration. Fertil Steril1996;65:280-87.

13. Chapron C, Dubuisson JB, Pansini V, Vieira M, Fauconnier A, Barakat $\mathrm{H}$, et al. Routine clinical examination is not sufficient for diagnosing and locating deeply infiltrating endometriosis. J Am Assoc Gynecol Laparosc 2002;9:115-19.

14. Dragisic KG, Padilla LA, Milad MP. The accuracy of the rectovaginal examination in detecting cul-de-sac disease in patients under general anaesthesia. Hum Reprod 2003;18:1712-15.

15. Bazot M, Detchev R, Cortez A, Amouyal P, Uzan S, Darai E. Transvaginal sonography and rectal endoscopic sonography for the assessment of pelvic endometriosis: a preliminary comparison. Hum Reprod 2003;18:1686-92.

16. Bazot M, Thomassin I, Hourani R, Cortez A, Darai E. Diagnostic accuracy of transvaginal sonography for deep pelvic endometriosis. Ultrasound Obstet Gynecol 2004;24:180-85.

17. Redwine DB. Ovarian endometriosis: a marker for more extensive pelvis and intestinal disease. Fertil Steril 1999;72:310-15.

18. Bazot M, Darai E, Hourani R, Thomassin I, Cortez A, Uzan S, et al. Deep pelvic endometriosis: MR imaging for diagnosis and prediction of extension of disease. Radiology 2004;232:379-89.

19. Landi S, Barbieri F, Fiaccavento A, Mainardi P, Ruffo G, Selvaggi $\mathrm{L}$, et al. Preoperative double-contrast barium enema in patients with suspected intestinal endometriosis. J Am Assoc Gynecol Laparosc 2004;11:223-28.

20. Donnez J, Pirard C, Smets M, Jadoul P, Squifflet J. Surgical management of endometriosis. Best Pract Res Clin Obstet Gynaecol 2004;18:329-48.

21. Yap C, Furness S, Farquhar C. Pre and post operative medical therapy for endometriosis surgery. Cochrane Database Syst Rev
2004;3:CD003678.

22. Urbach DR, Reedijk M, Richard CS, Lie KI, Ross TM. Bowel resection for intestinal endometriosis. Dis Colon Rectum1998;41:1158-64.

23. Donnez J, Nisolle M, Squifflet J. Ureteral endometriosis: a complication of rectovaginal endometriotic (adenomyotic) nodules. Fertil Steril 2002;77:32-7.

24. Duepree HJ, Senagore AJ, Delaney CP, Marcello PW, Brady KM, Falcone T. Laparoscopic resection of deep pelvic endometriosis with rectosigmoid involvement. J Am Coll Surg 2002;195:754-58.

25. Jerby BL, Kessler H, Falcone T, Milsom JW. Laparoscopic management of colorectal endometriosis. Surg Endosc 1999;13:1125-28.

26. Ribeiro PA, Rodrigues FC, Kehdi IP, Rossini L, Abdalla HS, Donadio N, et al. Laparoscopic resection of intestinal endometriosis: a 5-year experience. J Minim Invasive Gynecol 2006;13:442-46.

27. Redwine DB, Sharpe DR. Laparoscopic segmental resection of the sigmoid colon for endometriosis. J Laparoendosc Surg1991;1:21720.

28. Selak V, Farquhar C, Prentice A. Danazol for pelvic pain associated with endometriosis. Cochrane Database Sys Rev 2007; 75:485-8.

29. Dousset B, Leconte M, Borghese B, Millischer A, Roseau G, Arkwright $\mathrm{S}$, et al. Complete surgery for low rectal endometriosis: Long-term results of a 100-case prospective study. Ann Surg 2010;251:887-95

30. Moawad NS, Guido R, Ramanathan R, Mansuria S, Lee T. Comparison of laparoscopic anterior discoid resection and laparoscopic low anterior resection of deep infiltrating rectosigmoid endometriosis. JSLS 2011;15:331-38.

31. Busnelli V, Mentz R, Vaccaro C, Antelo Galarza R, Im V, Gil S, et al. tratamiento laparoscópico resectivo por endometriosis severa con compromiso colorrectal: Factibilidad y seguridad. Rev Argent Coloproct 2014;25:204-10.

32. Renner SP, Kessler H, Topal H, Proske K, Adler W, Burghaus S, et al. Major and minor complications after anterior rectal resection for deeply infiltrating endometriosis. Arch Gynecol Obstet 2017;295:1277-85

33. Dousset B, Leconte M, Borghese B, Millischer AE, Roseau $\mathrm{G}$, Arkwright S, et al. Complete surgery for low rectal endometriosis: long-term results of a 100-case prospective study. Ann Surg 2010;251:887-95.

\section{COMENTARIO}

El trabajo que presenta el Dr. Zapata y colaboradores, del Hospital de Clínicas, destaca los pacientes operados por endometriosis en dicha institución. Debido a la gran variedad sintomática el diagnóstico suele ser tardío y puede alcanzar al 30-40\% de las mujeres con infertilidad. A pesar de los avances en los métodos diagnósticos el abordaje laparoscópico sigue siendo de elección. La experiencia radica en 171 pacientes intervenidos, de los cuales 29 presentaban patología colorrectal. Las tasas de conversión y de morbimortalidad son aceptables y acordes a la literatura internacional. Es interesante destacar la revisión actualizada del tema. Resulta lógico pensar que la gran cantidad de pacientes incluidos en la serie depende de un gran compromiso multidisciplinario, trabajando en conjunto con el servicio de ginecología y otros servicios pertinentes.

Mariano Laporte

Hospital Alemán. Ciudad Autónoma de Buenos Aires, Argentina. 